\title{
Prominence Science with ATST Instrumentation
}

\author{
Thomas Rimmele $^{1}$, Thomas Berger ${ }^{1}$, Roberto Casini ${ }^{2}$, David Elmore ${ }^{1}$, \\ Jeff Kuhn ${ }^{3}$, Haosheng Lin ${ }^{3}$, Wolfgang Schmidt ${ }^{4}$ and Friedrich Wöger ${ }^{1}$ \\ ${ }^{1}$ National Solar Observatory, Sunspot, NM-88349, \\ PO Box 62,USA \\ email: rimmele@nso.edu \\ ${ }^{2}$ High Altitude Observatory, Boulder, CO, \\ ${ }^{3}$ Institute for Astronomy, University of Hawaii, \\ ${ }^{4}$ Kiepenheuer Institute für Sonnenphysik, Freiburg, Germany
}

\begin{abstract}
The 4m Advance Technology Solar Telescope (ATST) is under construction on Maui, HI. With its unprecedented resolution and photon collecting power ATST will be an ideal tool for studying prominences and filaments and their role in producing Coronal Mass Ejections that drive Space Weather. The ATST facility will provide a set of first light instruments that enable imaging and spectroscopy of the dynamic filament and prominence structure at 8 times the resolution of Hinode. Polarimeters allow high precision chromospheric and coronal magnetometry at visible and infrared (IR) wavelengths. This paper summarizes the capabilities of the ATST first-light instrumentation with focus on prominence and filament science.
\end{abstract}

Keywords. Sun, Prominence, Filaments, Corona, Magnetic Fields, Instrumentation

\section{Introduction}

Prominences are elevated structures observed at the limb of sun. On the disk these features are seen as dark filaments in chromospheric lines such as $\mathrm{H} \alpha$. Prominences and filaments are large-scale structures of cool plasma embedded in an otherwise hot coronal environment. The cold prominence plasma is suspended by a complex magnetic fields. Prominences and filaments are drivers of space weather. The contribution by Gibson in this volume provides an overview of modeling efforts that describe the complex magnetic field configurations of prominences. Recent high-resolution time sequences of narrow band images exhibit intriguing, highly dynamic and variable properties (see e.g. contribution by Berger in this volume). These new data challenge conventional views of the nature of prominences. However, detailed diagnostics of the thermodynamic and magnetic properties of the prominences are still lacking due to the limitations of the imaging instruments used to obtain these observations.

The ATST with its $4 \mathrm{~m}$ aperture and a set of highly capable first light instruments offers unique capabilities for ground-breaking observations and quantitative analysis of prominences and filaments. The significantly increased resolution and the photon collecting area provided by the large aperture open up new discovery space. One of the most challenging goals of the ATST is to perform measurements of the prominence (filament) magnetic field and the coronal background field, in which the prominence is embedded. ATST instrumentation will provide the spectroscopic analysis tools that cover a wide temperature range and allow measurements of the chromospheric and coronal magnetic field with sufficient sensitivity. The high photon flux of ATST is essential to accurately measure Stokes-V, which provides crucial diagnostics needed to distinguish between magnetic field models, for example, between a potential field extrapolation and a non-linear force 


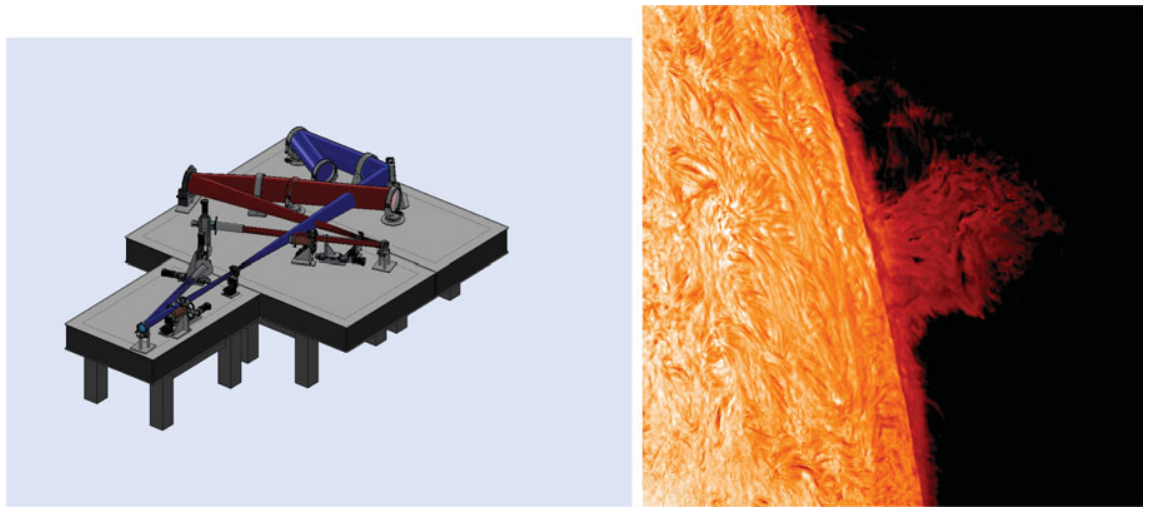

Figure 1. Right: VBI instrument layout. Left: Image of a prominence observed at the DST with the VBI $\mathrm{H} \alpha$ filter.

free extrapolation (Wiegelmann 2004). Spectral diagnostic in the infrared is particularly attractive for coronal spectroscopy and magnetometry due to low sky and instrumental background and relatively bright mid-IR coronal emission lines.

This paper reviews the design and capabilities of the ATST first light instruments. These instruments can be operated individually or as a system and will provide a wealth of observational information about prominences that promises to significantly advance our knowledge of these important drivers of space weather.

\section{ATST First Light Instruments}

The suite of five first light instruments consists of the Visible Broad-band Imager (VBI), the Visible Tunable Filter (VTF), the Visible Spectro-Polarimeter (ViSP), the Diffraction-Limited Near-Infrared Spectro-Polarimeter (DL-NIRSP) and the Cryogenic Near-Infrared Spectro-Polarimeter (Cryo-NIRSP). The VBI and the VTF provide broadband and narrowband imaging while the ViSP and the NIRSP instruments are spectrograph-based instruments. All instruments share a camera and data handling system provided by the facility. Polarimetry calibration and modulation are also functions of facility subsystems. Making adaptive optics function using the faint prominence structure that can be observed only with narrow-band $(0.5 \AA)$ filters (e.g. H $\alpha$ ) is a challenge. A prototype system has recently been tested successfully at the Dunn Solar Telescope (DST) (Taylor et al. 2013). The ATST instruments build on significant heritage of instruments operated at current facilities, such as the DST. The goal is to operate these instruments in Service Mode (Uitenbroek\& Tritschler 2012) for the majority of available observing time. The data collected by all instruments will be processed to remove instrument and residual atmospheric signatures (Level 1) at the NSO-ATST Data Center located at NSO Headquarters in Boulder. Distribution of data products for a broad user base is a function of the Data Center. The Science Working Group is defining standard data products for each of the instrument that will be available to the community.

\subsection{Visible Broadband Imager (VBI)}

The ATST Visible Broadband Imager (VBI) is a high-cadence imaging instrument that resolves structures within a $2 \times 2 \operatorname{arcmin}^{2}$ field in the solar photosphere and chromosphere at the diffraction limit of ATST's aperture. The VBI consists of two imaging channels that can be synchronized to simultaneously observe targets at two different wavelengths in the visible light wavelength range between 390 and $800 \mathrm{~nm}$ selected by interference 
Table 1. VBI channel passbands.

\begin{tabular}{|c|c|c|}
\hline VBI blue & VBI red & \\
\hline $393.3 \mathrm{~nm}$, FWHM $0.1 \mathrm{~nm}$ |chromosphere (Ca II K) & \multirow{2}{*}{\multicolumn{2}{|c|}{$656.3 \mathrm{~nm}$, FWHM $0.05 \mathrm{~nm} \mid$ chromosphere $(\mathrm{H} \alpha)$}} \\
\hline $430.5 \mathrm{~nm}$, FWHM $0.5 \mathrm{~nm}$ | photosphere (G-band) & & \\
\hline $450.4 \mathrm{~nm}$, FWHM $0.4 \mathrm{~mm} \quad$ |photosphere (blue continuum) & $668.4 \mathrm{~nm}$, FWHM $0.4 \mathrm{~nm}$ & photosphere (blue continuum) \\
\hline $486.1 \mathrm{~nm}$, FWHM $0.05 \mathrm{~nm}$ |chromosphere $(\mathrm{H} \beta)$ & $705.8 \mathrm{~nm}$, FWHM $0.6 \mathrm{~nm}$ & photosphere $(\mathrm{TiO})$ \\
\hline
\end{tabular}

filters as narrow as $0.05 \mathrm{~nm}$ for its chromospheric lines. The main science performance requirements of the VBI are: 1) temporal cadence of 3.2 seconds between reconstructed, high-resolution images observed at any selected wavelength in the same channel 2) critical sampling (or better) of ATST's diffraction limit at wavelengths $>400 \mathrm{~nm} 3$ ) imaging of eight prominent lines and bands covering the photosphere and chromosphere, with high throughput.

For performance reasons, the VBI channel wavelengths are separated between 'blue' and 'red' wavelengths. Both channels contain interference filters that image either photospheric or chromospheric layers in the solar atmosphere. Table 1 lists the available interference filters and their passband.

The high-resolution, high-cadence capabilities of the two channels of the VBI will allow recording simultaneous images of, e.g., the chromospheric Ca II K (393.3 nm) line and the $\mathrm{H} \alpha(656.3 \mathrm{~nm})$ line (see Fig. 1) to investigate the dynamics and morphology of prominences within multiple layers of the solar atmosphere. These well-known and common diagnostics for prominence observations can be complemented by reconstructed images of the $\mathrm{H} \beta(486.1 \mathrm{~nm})$ line that might allow to infer further information about the temperature characteristics of the prominence. The ability to switch to any wavelength combination achievable by the interference filter configuration in each channel, and to acquire two simultaneous, reconstructed high-spatial resolution images at a fixed cadence enables accurate measurements of oscillations on-disk, or proper motions of prominence fine structure through the different geometric heights at the limb. In addition, the movies created will deliver important context for ATST instruments that have spectro-polarimetric capabilities but either operate at lower spatial resolution or within a smaller field of view.

\subsection{Visible Tunable Filter (VTF)}

The VTF is an imaging spectro-polarimeter based on tunable Fabry-Perot Etalons. It can observe virtually all spectral lines (one at a time) within the wavelength range of the instrument. It produces narrowband filtergrams with a field of view of about one arc minute. Several spectral lines can be observed in rapid sequence, where the cadence ranges between $1 \mathrm{~s}$ and $30 \mathrm{~s}$ per line, depending on the spectral sampling and on the desired signal quality. The VTF offers the several measurement modes:

- Imaging Stokes Polarimeter to derive magnetic field information from the polarization properties of spectral lines. This mode includes the possibility to measure full Stokes data set to retrieve the magnetic field, or to record Stokes-V magnetograms.

- Doppler imager to measure flow fields in the solar atmosphere

- Narrowband filter imager to record monochromatic filtergrams

The VTF covers a spectral range from $520 \mathrm{~nm}$ to $870 \mathrm{~nm}$, with a spectral resolution of $3 \mathrm{pm}(\mathrm{R}=185,000$ at $550 \mathrm{~nm})$ and a circular field of view of 60 arcsec. The optical design uses three Fabry-Perot Etalons with spacing ratios of 1:1.581:3.412 in a telecentric configuration. The VTF has a pixel scale of 0.0125 arcsec and thus allows for diffractionlimited imaging with the ATST. The integration time for individual images is $25 \mathrm{~ms}$, 

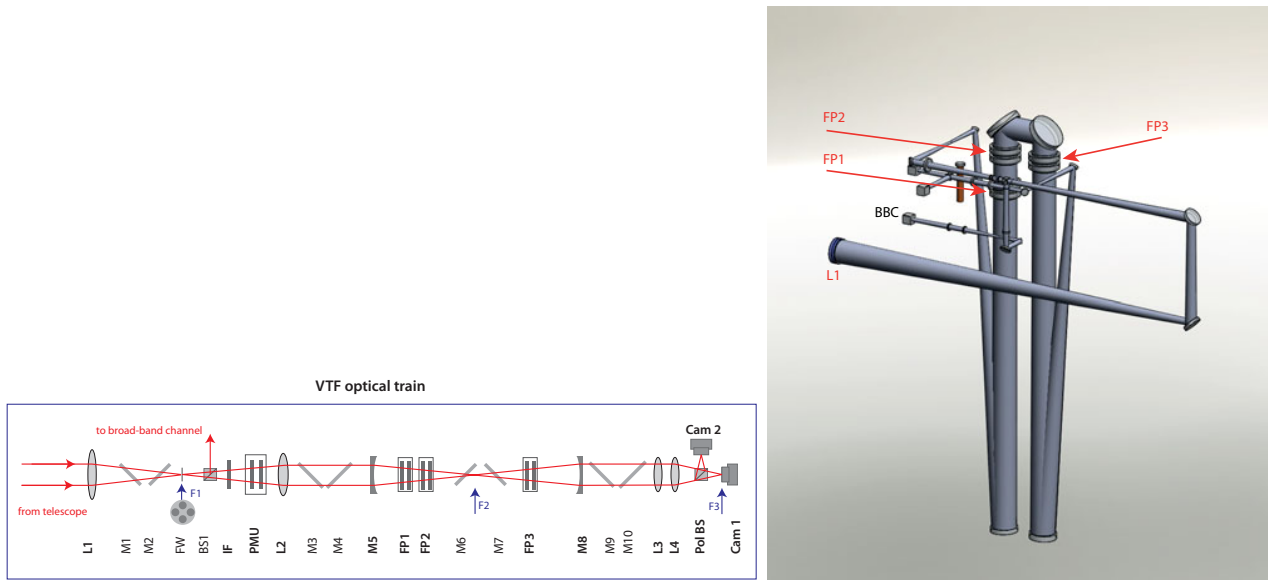

Figure 2. Left: Optical train of the VTF. Lenses and mirrors are labeled with Lx and Mx, respectively. FW is the device with the line selecting filters, PMU is the polarization modulation unit. The three Etalons are labelled FP1,2,3. A polarizing beam splitter (PolBS) serves as analyzer and sends two polarized beams to the detectors, Cam 1 and Cam2. Right: Optical configuration of the VTF. The three Etalons are marked in red $(F P 1,2,3)$. The broad-band channel is labeled $B B C$. Lens $L 1$ images the field of view to the VTF entrance focal plane.

corresponding to a signal-to-noise of 90 at disk center. The signal quality is improved through accumulating several images per wavelength position per Stokes component. Depending on the strength of the line and on the number of image accumulations, a signal-to noise-ratio of 300 is achieved. The spectral field of view can be chosen to cover line shifts up to $50 \mathrm{kms}^{-1}$. Line selection is done with interference filters with a width of about $1 \mathrm{~nm}$. The optical train of the VTF is shown in Fig.2, left. It shows the optical elements of the narrow-band channel. In the opto-mechanical configuration (Fig. 2,right) the Etalons will be placed horizontally, i.e. with the light beam passing vertically, to minimize gravitational deformation of the air gap between the Etalon plates. The Etalons for the VTF will have clear apertures of $260 \mathrm{~mm}$.

The spectral window of the VTF includes the Mg b lines, the He D3 line, the Na D lines, $\mathrm{H} \alpha$, and the infrared Calcium lines. All these lines have been used to study the NLTE behavior of the prominence atmosphere (Stellmacher\& Wiehr 2005; Stellmacher et al. 2013). The VTF is therefore well suited for diagnostic spectroscopy of prominences, however with the restriction that only one line at a time can be recorded. In quiescent prominences, the cadence of multiline observations should be fast enough to combine the information obtained in different lines. The VTF records an area of about $30,000 \mathrm{~km} \times 30,000 \mathrm{~km}$ instantaneously at each wavelength point. Magnetic fields estimates should be possible from He D3 Stokes spectra. Due to the low intensity of prominences compared to on-disk measurements, the quality of the Stokes measurements will be significantly reduced. It may be necessary to sacrifice some spatial resolution to improve the sensitivity.

\subsection{Visible Spectro-Polarimeter (ViSP)}

The Visible Spectro-Polarimeter (ViSP) of the ATST is a wavelength versatile instrument, whose design concept is specifically to provide a continuous coverage of the instrument's spectral range of operation (380 to $900 \mathrm{~nm}$ ) over a field of view of $2 \times 2 \mathrm{arcmin}^{2}$. The main science performance requirements of the ViSP are: 1) spectral resolution better than $180,000(\sim 3.5 \mathrm{pm}$ at $630 \mathrm{~nm})$ over the entire spectral range; 2$)$ spatial (sampling) resolution matching the diffraction limit of the ATST at the wavelength of observation; 
3) temporal resolution of $10 \mathrm{~s}$ for spectro-polarimetric observations achieving $10^{-3}$ polarimetric sensitivity (strongly dependent on the target's brightness).

In order to approach as closely as possible the required spatial sampling condition at the observed wavelength, the ViSP is equipped with three photo-etched slit apertures, matching the Airy radius of the ATST respectively at 450,650, and $850 \mathrm{~nm}$. The ViSP is capable to observe up to three different spectral lines simultaneously. In order to do this, the ViSP implements low-order, high-dispersion diffraction gratings, and three movable spectral arms to pick up the different diffracted wavelengths (Fig. 3, right). Due to evident limits of geometric configurability, and depending on the desired performance of a specific observation, not all possible combinations of three wavelengths within the spectral range of operation of the ViSP may be attainable by the instrument. Nonetheless, the spectral diversity of the ViSP is significant, and allows the observations of many interesting pairs or triplets of solar lines, with good enough efficiency to meet the ATST science requirements.

The ViSP instrument covers the shorter end of the spectral region accessible to the ATST. This reaches down to $380 \mathrm{~nm}$ on the blue end, in order to allow spectropolarimetric observations of the strong chromospheric resonance lines of Ca II at $393.4 \mathrm{~nm}$ (K line) and $396.9 \mathrm{~nm}$ ( $\mathrm{H}$ line). On the red end of the "visible" solar spectrum, the ViSP spectral coverage extends to $900 \mathrm{~nm}$, thus encompassing also the Ca II IR triplet $(\lambda \lambda$ $849.8,854.2,866.2 \mathrm{~nm}$ ). The ViSP optics are optimized up to $1.1 \mu \mathrm{m}$, and so observations of the bright chromosperic resonance line of He I at $1083.0 \mathrm{~nm}$ are also possible, although the high quantum efficiency of a dedicated IR detector may be necessary to perform high-sensitivity spectro-polarimetric observations of this line with the ViSP. The Ca II $\mathrm{H}$ and $\mathrm{K}$ resonance lines are among the strongest spectral features of the quiet-Sun spectrum, and they represent important tracers of small-scale magnetic activity in the solar atmosphere. They have also been regularly observed in solar prominences for more than a century (Belopolsky 1908; Berger et al. 2010), and through an accurate wavelength calibration of spectral observations they allow high precision Doppler measurements of prominence plasma motions. Theoretical studies of the NLTE formation of Ca II and H I lines in prominences Gouttebroze, Vial \& Heinzel (1997) have suggested that line-ratio techniques applied to various lines of these two different ion species can reliably be used for temperature diagnostics of prominence plasmas (Gouttebroze \& Heinzel 2002).

For the magnetic diagnostics of prominences, the two chromospheric lines of He I at 587.6 (D3) and $1083.0 \mathrm{~nm}$ represent an almost ideal combination, because of the sensitivity of their polarization to a large range of magnetic strengths(approximately, 1 to $10^{3}$ gauss). This is possible because of the diverse diagnostic capabilities that are offered by the Hanle, Zeeman, and Paschen-Back effects of helium (Bommier 1980; Landi Degl'Innocenti 1983; López Ariste \& Casini 2002; Asenio Ramos et al. 2008). Indeed, these two lines have almost exclusively dominated all attempts of the solar scientific community to reliably measure the magnetic field of quiescent and active-region prominences and filaments (Leroy 1981; Querfeld et al. 1985; Bommier et al. 1994; Paletou 2001; Trujillo-Bueno et al. 2002; Casini et al. 2003; Kuckein et al. 2009). Based on both line formation modeling and the reported observations described above, a potential list of interesting solar lines for prominence diagnostics accessible with the ViSP is proposed in Table 3.

\subsection{Diffraction-Limited Near-Infrared Spectro-Polarimeter (DL-NIRSP)}

Unlike other ATST spectropolarimeters based on the conventional slit spectrograph design, DL-NIRSP will be equipped with two fiber-optic integral field units (IFUs) that will allow it to record the polarized spectra of a $2 \mathrm{D}$ field simultaneously in up to three 


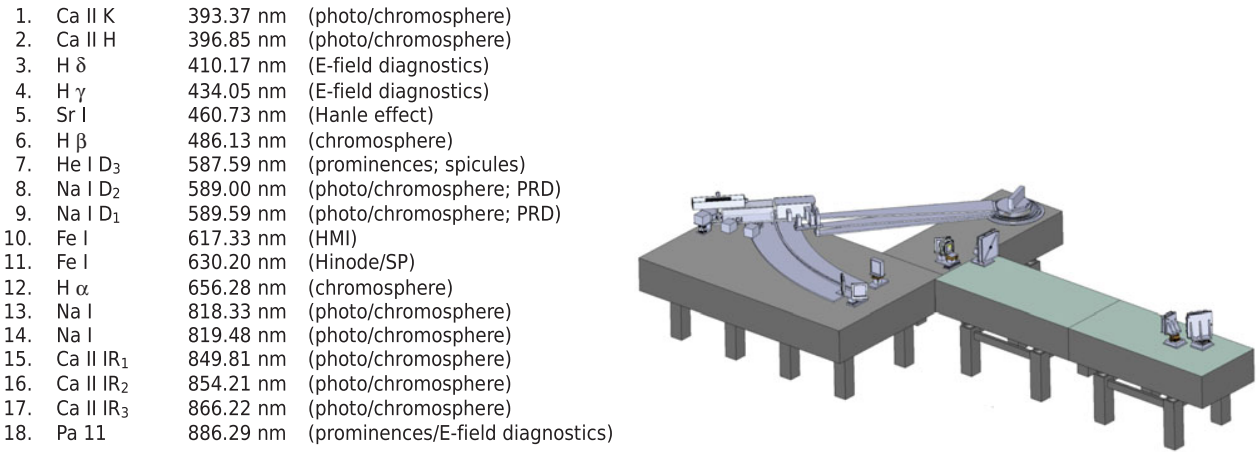

Figure 3. Left: Sample prominence diagnostics accessible with the ViSP. PRD stands for partial re-distribution. Right: Design of the Visible Spectro-Polarimeter (VISP).

spectral lines. This approach will enable observations of the dynamic evolution of the gas and magnetic fields of the prominences with high spatial and temporal resolution while also retaining the full spectral diagnostic capabilities of the conventional spectrographs. The DL-NIRSP will be equipped with a feed optical system that provides three different image scales, three spectral arms, and two diffraction gratings to provide a very versatile observing capability that can meet the observing requirements of programs with disparate science objectives. The DL-NIRSP can also observe three spectral lines simultaneously, with flexible line combination, which will provide the data needed for comprehensive spectral diagnostic to fully explore the prominence atmosphere in a broad temperature, density, and magnetic field ranges. This instrument allows to perform trades between spatial (and temporal) resolution and sensitivity. Figure 4, right, shows the field of view of DL-NIRSP in three observing modes: 1$)$ a 7.2 " $\times 5.4$ " $(0 " .03 /$ pix $)$ field obtained with a $9 \times 9$ mosaic using the $\mathrm{F} / 62$ high-resolution mode, 2) a 19.2" $\times 14.4 "(0 " .08 /$ pix $)$ field obtained with a $3 \times 3$ mosaic in the F/24 mid-resolution mode, and 3) a $80 " \times 60$ " field with 1" spatial sampling without mosaic. The IFU Imaging Array Format is $80 \times 60$. The spectral resolution $\frac{\lambda}{\Delta \lambda}$ is 250,000 (F/62, F24) and 80,000 (F/4). Figure 4, left, shows the optical layout of the 3 -arm DL-NIRSP spectrograph.

DL-NIRSP will split the solar spectrum into three wavelength windows. The first window covers the spectral range of 500 to $750 \mathrm{~nm}$, the second spectral windows covers the range of $750 \mathrm{~nm}$ to $1,000 \mathrm{~nm}$, and the third windows covers $1,000 \mathrm{~nm}$ to the cutoff
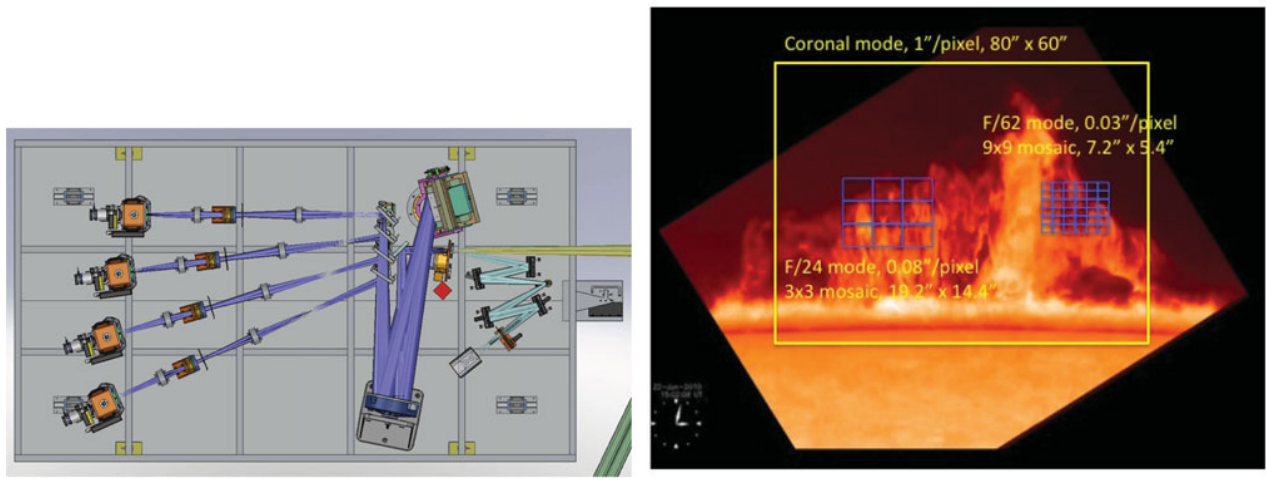

Figure 4. Left: optical design of DL-NIRSP. Right: FOV of DL-NIRSP for different feed optics configurations. 
wavelength of the IR array detector. Table 2 shows some possible line combinations, limited by the availability of bandpass isolation filters for the spectral lines.

Table 2. DL-NIRSP possible Line Combinations

\begin{tabular}{|l||c|c|c|}
\hline & Spectral Arm $1(500-750 \mathrm{~nm})$ & Spectral Arm 2 $(750-1,000 \mathrm{~nm})$ & Spectral Arm $3(1,000-2,500 \mathrm{~nm})$ \\
1 & H $2656.3 \mathrm{~nm}$ & CaII $854.2 \mathrm{~nm}$ & HeI $1083.0 \mathrm{~nm}$ \\
2 & HeI D3 $587 \mathrm{~nm}$ & CaII $854 \mathrm{~nm}$ & HeI $1083 \mathrm{~nm}$ \\
3 & HeI D3 $587 \mathrm{~nm}$ & CaII $854 \mathrm{~nm}$ & FeXIII $1075 \mathrm{~nm}$ \\
\hline
\end{tabular}

\subsection{Cryogenic Near-Infrared Spectro-Polarimeter (Cryo-NIRSP)}

The ATST and its IR instrumentation bring spectacular advantages for observing prominences because of its low-scattered light performance and broad wavelength coverage. Off-limb prominence observations depend on the suppression of scattered light from the solar disk and a reduced sky background. The ATST achieves both goals because of its dark-sky operating conditions from Haleakala and its all-reflecting coronagraphic optical design. While the ATST field-of-view is limited to about 4', this is adequate for observations of most off-limb magnetic structures. The extended wavelength coverage of the ATST allows instrumentation that can further improve the scattered light dynamic range of prominence measurements. The sky brightness decreases with wavelength. In particular, in the wavelength window between 3 and 4 microns that the terrestrial "blue" sky can be even fainter than the solar corona. Instrumental scattered light also declines in the IR, for example from mirror microroughness, where the background "noise" brightness decreases like $1 / \lambda^{2}$.

\begin{tabular}{|l|c|}
\hline Grating (primary) & Echelle: 32 line $/ \mathrm{mm}$ \\
Relay, focal ratio & 18 \\
Coll/Cam. Focal Ratio & $18 / 8$ \\
Plate scale & $0.16 \mathrm{~mm} /$ arcsec \\
Slit Width & $52 / 175 \mu$ \\
Pixel scale (at 1 $\mu)$ & $165 \mathrm{~m} \AA$ \\
Pixel scale (arcsec) & $0.12 "(18 \mu$ pix) \\
Design/Diffraction limited FOV & $240 / 90 "$ \\
Cold blocking filter & BW $1 \%$ \\
System QE & $5 \%$ \\
Max spectral resolution at $4 \mu$ & 100000 \\
\hline
\end{tabular}

Table 3. CryoNIRSP Optical

Specifications

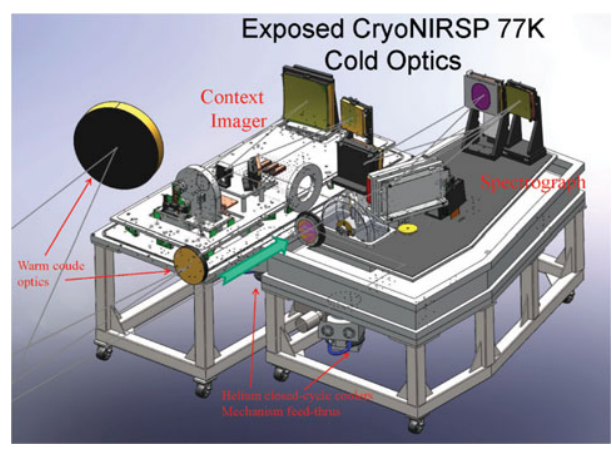

Table 4. The CryoNIRSP optics are cooled to approximately $80 \mathrm{~K}$ in order to achieve photon-noise limited performance from visible to IR wavelengths of $5 \mu$ for bright and faint infrared sources.

Prominence magnetometry depends on very high signal-to-noise spectropolarimetry. For example the important HeI IR triplet-state resonance lines will be critical for both permitted-line, non-saturated linear polarization Hanle measurements and the even more difficult Stokes-V magnetometry of off-limb prominence magnetic fields. These studies depend on the high dynamic range capabilities of ATST and its post-focus instrumentation. The high-dynamic range and wide-field IR spectropolarimetry that ATST and its instrumentation will deliver should significantly improve prominence vector magnetometry with a sensitivity that can exceed $0.1 \mathrm{G}$.

The CryoNIRSP imaging spectropolarimeter is designed to take advantage of the ATST's unmatched scattered light suppression, broad wavelength coverage, and fieldof-view. It is thus a fully reflective, essentially photon-limited spectropolarimeter that 
observes over a 0.5 (goal) to $5 \mu$ wavelength band. It is designed to take full advantage of the ATST's coronagraphic properties while providing slit-scanning spectropolarimetry of a 3'x4' field with a spectral resolution of 100,000. To achieve high polarimetric accuracy it simultaneously observes orthogonal polarization states using a cryogenic polarizing beamsplitter. CryoNIRSP is the largest FOV ATST instrument and does not use system AO facilities. Under good seeing conditions the ATST/CryoNIRSP will be diffraction limited near $4.6 \mu$ wavelengths. To achieve photon-noise limited performance the essential instrument optics and detectors are cryogenically cooled. Instrument properties are summarized in Table 3.

The CryoNIRSP context imager and spectrograph can be operated independently for observations of the near-limb or far-limb solar atmosphere. Both modes are well suited to observing the origin and evolution of cool magnetic prominence loops into the hot coronal surroundings. Figure 4 shows how the optical components of the instrument will appear at the coude level of the ATST with dewar thermal shields removed. The instrument's wide FOV means it is uniquely suited for both Zeeman and Hanle effect prominence vector magnetometry using, for example, the IR Helium lines near $1 \mu$. The system's low scattered light performance allows direct measurement of the imbedding coronal magnetic field that is critical to understanding the stability and evolution of the cooler prominence material. This is possible because the CryoNIRSP can measure $1 \mathrm{G}$-level coronal fields over a broad range of plasma temperatures. For this it relies on the sensitivity of forbidden coronal emission lines ranging in wavelength from the green line $(0.53 \mu)$ to $\mathrm{SiX}$ near $4 \mu$ where the magnetic Zeeman sensitivity is also a maximum.

\section{References}

Wiegelmann, T. 2004, Sol. Phys., 219, 87

Uitenbroek, H. \& Tritschler, A. 2012, IAU Special Session, 6

Taylor, G. et al., 2013, Proceddings SPIE, Optics and Photonics 2013: Astronomical Optics and Instruments, in press.

Stellmacher, G. \& Wiehr, E. 2005, Astron. Astrophys., 431, 1069

Stellmacher, G., Wiehr, E., \& Dammasch, I. E. 2013, arXiv:1303.1126

Asensio Ramos, A., Trujillo Bueno, J., \& Landi Degl'Innocenti, E. 2008, ApJ, 683, 542

Belopolsky, A. A. 1908, MiPul (Bull. Pulkovo Obs.), 2, 239

Berger, T. E., Slater, G., Hurlburt, N., et al. 2010, ApJ, 716, 1288

Bommier, V. 1980, Astron. Astrophys., 87, 109

Bommier, V., Landi Degl'Innocenti, E., Leroy, J.-L., \& Sahal-Bréchot,S. 1994, Solar Phys., 154, 231

Casini, R., López Ariste, A., Tomczyk, S., \& Lites, B. W. 2003, ApJ, 582, 51L

Gouttebroze, P. \& Heinzel, P. 2002, Astron. Astrophys., 385, 273

Gouttebroze, P., Vial, J.-C., \& Heinzel, P. 1997, Solar Phys., 172, 125

Kuckein,C., Centeno, R., Martínez Pillet, V., et al. 2009, Astron. Astrophys., 501, 1113

Landi Degl'Innocenti, E. 1982, Solar Phys., 79, 291

Leroy, J.-L. 1981, Solar Phys., 71, 285

López Ariste, A., \& Casini, R. 2002, ApJ, 575, 529

Paletou, F., López Ariste, A., Bommier, V., \& Semel, M. 2001, Astron. Astrophys., 375, 39

Querfeld, C. W. , Smartt, R. N. , Bommier, V., et al. 1985, Solar Phys., 96, 277

Trujillo Bueno, J., Landi Degl'Innocenti, E., Collados, M., et al. 2002, Nature, 415, 403 